\title{
Myocarditis related to immune checkpoint inhibitors treatment: two case reports and literature review
}

\author{
Yukai Chen $^{1 \#}$, Yijun Jia ${ }^{1 \#}$, Qinghua Liu ${ }^{1}$, Yunli Shen ${ }^{2}$, Hui Zhu ${ }^{1}$, Xue Dong ${ }^{1}$, Jianhao Huang ${ }^{1}$ Jingjing Lu ${ }^{1}$, \\ Qi Yin ${ }^{1}$ \\ ${ }^{1}$ Department of Pulmonary and Critical Care Medicine, Shanghai East Hospital, Tongji University School of Medicine, Shanghai, China; \\ ${ }^{2}$ Department of Cardiology, Shanghai East Hospital, Tongji University School of Medicine, Shanghai, China \\ \#These authors contributed equally to this work. \\ Correspondence to: Jingjing Lu; Qi Yin. Department of Pulmonary and Critical Care Medicine, Shanghai East Hospital, Tongji University School of \\ Medicine, Shanghai, China. Email: lujjhf@163.com; yvonneyinqi2019@126.com.
}

\begin{abstract}
Immune checkpoint inhibitors can cause immune-related toxicity in various systems, and myocarditis is the most serious life-threatening toxicity. This report introduces diagnosis and treatment of two cases which developed myocarditis after receiving PD-1 inhibitors therapy. The first case was a 77-yearold male with chordoma, who was treated by third-line sintilimab combined with anlotinib, and presented with symptoms of chest tightness, shortness of breath and upper eyelid ptosis three weeks later. He was diagnosed as immune-checkpoint-inhibitors-related myocarditis and myositis-myasthenia-gravis overlap syndrome based on his clinical symptoms, serum biomarkers, electrocardiogram, echocardiogram, and characteristic findings on cardiac 18F-FDG PET-MRI. Methylprednisolone was given $480 \mathrm{mg} / \mathrm{d}$ initially and was gradually reduced to $40 \mathrm{mg} / \mathrm{d}$ in 4 weeks, with the myocardial injury biomarkers declined in the same time. The second case was a 69 -year-old female with advanced non-small cell lung cancer, who was treated by pemetrexed combined with bevacizumab and camrelizumab, and presented with palpitations 20 days later. She was diagnosed as immune-checkpoint-inhibitors-related myocarditis based on her clinical symptoms, serum biomarkers, electrocardiogram and echocardiogram. Methylprednisolone was given $240 \mathrm{mg} / \mathrm{d}$ initially and was gradually reduced to $40 \mathrm{mg} / \mathrm{d}$ with good response of myocardial injury biomarkers decline. However, the patient developed fatal myasthenia gravis afterwards, with little response to all treatments. These two cases revealed that, early detection and timely intervention, including discontinuation of immune checkpoint inhibitors and initiation of adequate steroid therapy, can reduce morbidity and mortality and improve prognosis.
\end{abstract}

Keywords: Immune checkpoint inhibitor; toxicity; immunotherapy-related adverse reactions; myocarditis; case report

Submitted Dec 29, 2020. Accepted for publication Mar 21, 2021.

doi: 10.21037/apm-20-2620

View this article at: https://dx.doi.org/10.21037/apm-20-2620

\section{Introduction}

By promoting $\mathrm{T}$ cell-mediated recognition and elimination of tumor cells, immune checkpoint inhibitors (ICIs) have dramatically improved clinical outcomes in various malignances (1). However, T cell activated by ICIs not only targets tumor cells but also induces autoimmune toxicities. Diverse immune-related adverse events (irAEs) involving pulmonary, cardiovascular, neurological, endocrine, gastrointestinal, and renal system can occur out of ICIs treatment (2). Myocarditis is a rare but potentially life-threatening complication of treatment with ICIs (3). ICIs associated myocarditis accounting for $1.14 \%$ of irAEs, but was correlated with a mortality of $46 \%$ (4). Besides, this condition can overlap with myositis or myasthenia gravis in more than $30 \%$ of patients receiving ICIs 

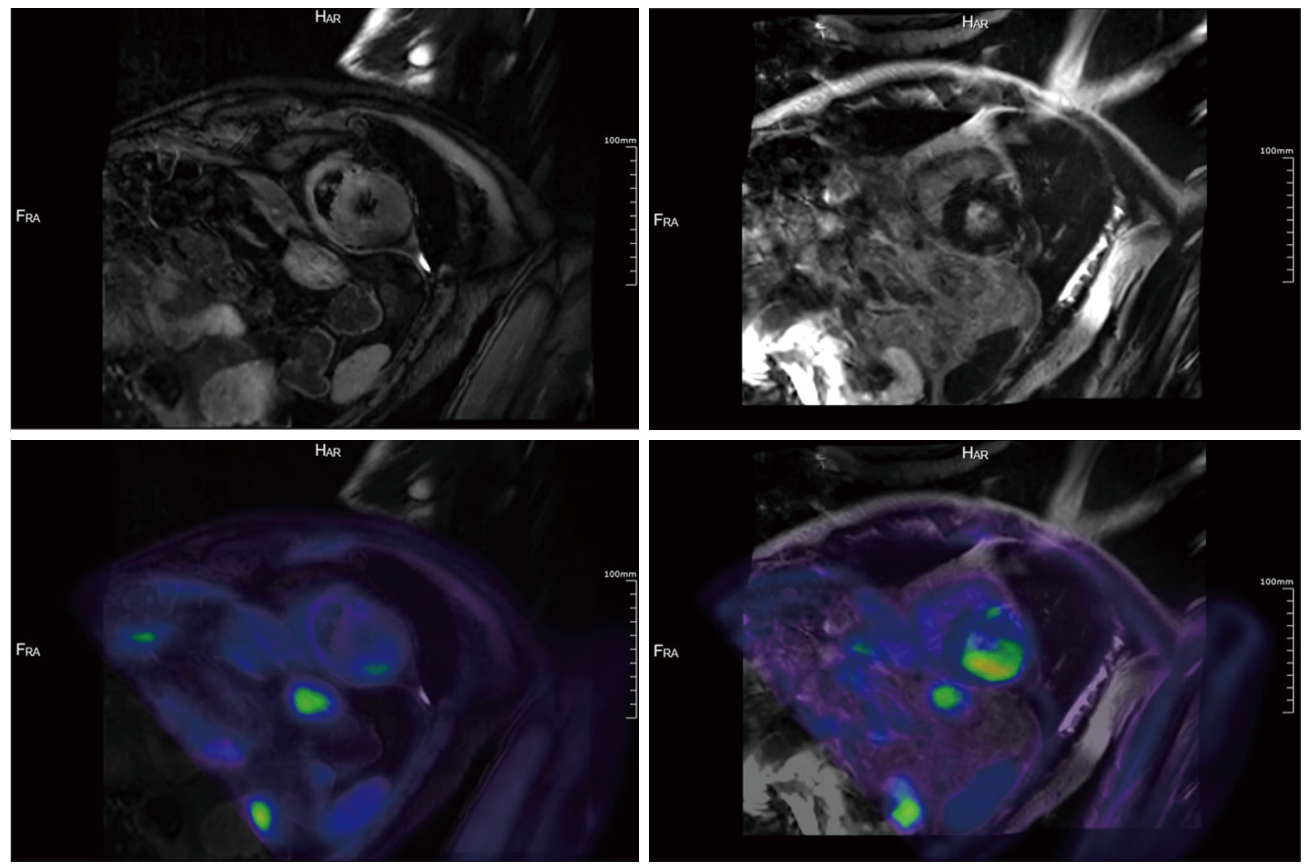

Figure 1 Position emission tomography magnetic resonance imaging (PET-MRI) demonstrated increased fluorodeoxyglucose (FDG) metabolism at the base of the left ventricular wall. The high signal area can be seen on T2-weighted imaging (T2WI).

treatment (5). In this report, we described a case of lung adenocarcinoma and a case of chordoma, both of which developed myocarditis after receiving PD-1 inhibitors therapy. We present the following article in accordance with the CARE reporting checklist. Available at: http:// dx.doi.org/10.21037/apm-20-2620.

\section{Case presentation}

\section{Case 1}

A 77-year-old male with chordoma was treated by thirdline sintilimab, a PD-1 antibody, combined with anlotinib, a tyrosine kinase inhibitor that targets VEGFR, FGFR, PDGFR and c-kit. At that time, he was evaluated as progressive disease after first line radiotherapy and second line treatment of anlotinib. He did not have any autoimmune disease or heart disease before.

The patient presented with symptoms of chest tightness, shortness of breath and upper eyelid ptosis three weeks after receiving his first dose of combination treatment. Physical exam was notable for bilateral upper eyelid ptosis, ocular motility limitation and lower limb weakness. Electrocardiogram (ECG) showed ST segment depression in leads I, II, aVF, V4, V5, and V6, with T wave changes in leads I and aVL. Echocardiogram revealed a left ventricular ejection fraction (LVEF) of $61 \%$ (normal value $>50 \%$ ) with an enlarged left atrium, and decreased left ventricular diastolic function. Cardiac troponin $\mathrm{T}$ (cTnT) level was $1.29 \mathrm{ng} / \mathrm{mL}$ (normal value $<0.014$ ) and rose to $1.5 \mathrm{ng} / \mathrm{mL}$ 6 hours later. N-terminal pro-brain natriuretic peptide (NT-proBNP) level was $581.8 \mathrm{ng} / \mathrm{L}$ (normal value $<125$ ). Creatine kinase-myocardial band (CK-MB) and creatine kinase (CK) levels were $140.7 \mathrm{ng} / \mathrm{mL}$ (normal value <4.87) and $706 \mathrm{U} / \mathrm{L}$ (normal value <310), respectively. Aspartate aminotransferase (AST) and alanine aminotransferase (ALT) levels were $403 \mathrm{U} / \mathrm{L}$ (normal value $<40$ ) and $168 \mathrm{U} / \mathrm{L}$ (normal value <50), respectively. Anti-acetylcholine receptor antibody (anti-AChR-Ab) level was $0.743 \mathrm{nmol} / \mathrm{L}$ (normal value $<0.45$ ) and anti-Titin body level was $1.269 \mathrm{nmol} / \mathrm{L}$ (normal value $<0.5$ ), respectively. Cardiac $18 \mathrm{~F}$-fluorodeoxyglucose (18F-FDG) position emission tomography magnetic resonance imaging (PET-MRI) demonstrated increased FDG metabolism at the base of the left ventricular wall and increased T2WI signals at the base of the ventricular septum (Figure 1). Based on his clinical symptoms, elevation of myocardial injury biomarkers, changes in ECG and echocardiogram, and characteristic findings on cardiac 18F-FDG PET-MRI, he was diagnosed 
with ICIs-related myocarditis. Immune-related hepatitis also occurred on this patient. Myositis-myasthenia gravis overlap syndrome was also highly suspected based on the symptoms of upper eyelid ptosis and elevated acetylcholinereceptor antibodies.

An immunosuppressive therapy of $480 \mathrm{mg} / \mathrm{d}$ methylprednisolone was given immediately for five days. The dose of methylprednisolone was gradually reduced to $40 \mathrm{mg} / \mathrm{d}$ in 4 weeks, with the CK and cTnT levels declined in the same time. Despite the good response to immunosuppression, the patient subsequently developed abdominal infection after 15 days of methylprednisolone treatment. The patient refused further intervention and requested to be discharged. He gave up all treatment and died at home three days later.

\section{Case 2}

A 69-year-old female with past medical history of arterial hypertension was diagnosed with advanced non-small cell lung cancer with EGFR/ALK wild-phenotype and was treated with pemetrexed combined with bevacizumab as first-line therapy. Camrelizumab, an anti-PD-1 monoclonal antibody, was added after two cycles of chemotherapy.

The patient presented with palpitations 20 days after the camrelizumab treatment. ECG showed sinus tachycardia, atrial premature beats, atrial tachycardia and ST segment depressed in leads I, II and aVF. Echocardiogram showed a LVEF of 59\% with enlarged inner diameter of the left atrium, decreased left ventricular diastolic function and normal left ventricular systolic function. cTnT level was $0.806 \mathrm{ng} / \mathrm{mL}$ (normal value $<0.014$ ), and rose to $0.952 \mathrm{ng} / \mathrm{mL}$ the next day. NTproBNP and CK-MB levels were $321 \mathrm{ng} / \mathrm{L}$ (normal value $<125$ ) and $81.8 \mathrm{ng} / \mathrm{mL}$ (normal value $<4.87$ ), respectively. AST and ALT levels were 339.4 U/L (normal value <40) and 144.2 U/L (normal value $<50$ ). The patient failed to do the cardiac MRI examination due to claustrophobia. A clinical diagnosis of immune-related myocarditis was made and were initiated with methylprednisolone at a dose of $240 \mathrm{mg} / \mathrm{d}$ for five days. The clinical symptoms improved and the myocardial injury biomarker levels declined, and the dose of methylprednisolone was decreased to $120 \mathrm{mg} / \mathrm{d}$ in the next two weeks. Subsequently the patient received $40 \mathrm{mg} / \mathrm{d}$ prednisolone orally and was discharged.

However, she was admitted for shortness of breath and progressive muscle weakness one week later. Physical examination found left upper eyelid ptosis and symmetrical weakness of the lower limbs. Initial arterial blood gas analyses demonstrated type II respiratory failure and noninvasive positive pressure ventilation (NIPPV) was applied. ECG and echocardiogram were the same as before. CK$\mathrm{MB}$ was $37.8 \mathrm{ng} / \mathrm{mL}$ (normal value $<4.87$ ) and $\mathrm{cTnT}$ was $0.175 \mathrm{ng} / \mathrm{mL}$ (normal value $<0.014$ ), which was same as before. NT-proBNP rose to $2,509 \mathrm{ng} / \mathrm{L}$ (normal value $<125)$. The thyroid hormones showed notably decreased free T3 of $0.88 \mathrm{pg} / \mathrm{dL}$ (normal value $0.80-2.00$ ) and free $\mathrm{T} 4$ of $0.60 \mathrm{ng} / \mathrm{dL}$ (normal value $0.93-1.70$ ), normal TSH level of $1.02 \mu \mathrm{IU} / \mathrm{mL}$ (normal value $0.27-4.20$ ). At the mean while the anti-thyroglobulin antibody and antithyroid peroxidase autoantibody were detected. $50 \mu \mathrm{g} / \mathrm{d}$ levothyroxine was prescribed and steroid treatment was changed to intravenous $40 \mathrm{mg} / \mathrm{d}$ methylprednisolone.

However, the weakness in respiratory muscles barely responded to the treatments. The patient was transferred to ICU due to the worsen carbon dioxide retention. AntiAChR-Ab was normal, but anti-Ryanodine-Receptor$\mathrm{Ab}$ and anti-Titin-Ab was positive. With suspicion of myasthenia gravis, the dose of methylprednisolone was adjusted to $120 \mathrm{mg} / \mathrm{d}$. Immunoglobulin was given intravenously for 15 days to prevent infections. Immunerelated myocarditis, hepatitis and hypothyroidism were improved, and methylprednisolone was weaned off over 3 weeks by tailing down $20 \mathrm{mg} / \mathrm{d}$ every week until a dose of $40 \mathrm{mg}$ daily was reached. Although the patient demonstrated a good response to steroid therapy initially, the weakness of respiratory muscle did not improve but progressed finally. She died of respiratory failure and pulmonary encephalopathy 52 days after her second admission.

All procedures performed in studies involving human participants were in accordance with the ethical standards of the institutional and/or national research committee(s) and with the Helsinki Declaration (as revised in 2013). Written informed consent was obtained from the patient.

\section{Discussion}

Herein we present a case of non-small cell lung cancer treated with camrelizumab and a case of chordoma treated with sintilimab, resulting in myocarditis concurrent with other irAEs. Different initial doses of methylprednisolone in two cases led to different outcomes, indicating that the initial dosage of methylprednisolone playing an important role in the prognosis of ICIs-related myocarditis. It was a pity that the first patient gave up further treatment for 
economic reasons, who was hopeful to get disease remission and live longer.

As ICIs are used widely to treat solid tumors, an increasing number of irAEs have been reported. Myocarditis is a one of the rare but fatal irAEs. The incidence of immune-related myocarditis is reported to be around 1\% (4), which is possibly under-estimated in the real world due to the challenging in diagnosis and a general lack of awareness of this condition (6). Because of the relatively low incidence, knowledge of the clinical manifestation and management of this condition is also limited.

These two patients in our report developed ICI-related myocarditis in an early period, after the first dose of ICIs treatment, which is consistent with prior studies $(4,5,7)$. Based on published reports, most ICI-related myocarditis occurred within the first three months of ICI therapy. Johnson et al. reported a median onset time of 17 days and a range of 13-64 days (8). In the study performed by Moslehi et al., myocarditis appeared after a median of 27 days after the initiation of immunotherapy, but with a wider range of 5-155 days (5). Both of our patients received a combined therapy of ICIs, one patient was treated with anti-angiogenesis therapy combined with PD-1 inhibitor, and the other received a chemotherapy and ICI combined treatment. It is reported that ICIs combination therapy is associated with a significantly higher incidence and mortality of myocarditis than monotherapy $(4,5)$. Other conditions like presence of cardiovascular risk factors or prior exposure to chest radiotherapy or agents with known cardiovascular toxicities may also increase the risk of ICIassociated cardiotoxicity $(4,5,7,9,10)$. Closer monitoring is needed especially for these patients.

The clinical manifestations of immune-related myocarditis are quite variable, which could range from nonspecific symptoms like fatigue, chest pain, or palpitation as the cases in our report, to heart failure and even sudden death. One typical feature of myocarditis is an elevated level of myocardial injury biomarkers such as $\mathrm{BNP}$ or NT-proBNP, cardiac troponin, and CKMB. ECG and echocardiogram were also helpful tools for initial evaluation (6). In our report, increased cardiac biomarkers and abnormality in ECG were presented in both patients. Although LVEF was normal, Immune-related myocarditis could not be ruled out. According to Mahmood et al. (4), over a half of patients diagnosed with Immunerelated myocarditis remained a normal LVEF. For now, there was no consensus of diagnosis criteria for immunerelated myocarditis. The application of endomyocardial biopsy, the gold-standard to diagnose myocarditis was limited for risk of severe complications, including fatal cardiac perforation. On the other hand, the pathological features of Immune-related myocarditis were sometimes similar with findings in acute allograft rejection in cardiac transplantation (8). Recently, 18F-FDG PET/MRI, which can provide both metabolic information and structural information by combing 18F-FDG PET and cardiac MRI in one study, is being increasingly recognized as a valuable tool in the detection of myocarditis $(11,12)$. Overall, a precise diagnosis of Immune-related myocarditis should involve a combination of clinical features, myocardial injury biomarkers and ECG, and/or endomyocardial biopsy if necessary. Emerging new procedures like PET/MRI may be beneficial to make the diagnosis.

Glucocorticoid is the first-line treatment for immunerelated myocarditis. For the patients with stable fatal signs, oral or intravenous prednisone $(1-2 \mathrm{mg} / \mathrm{kg} / \mathrm{d})$ is recommended (13). If the patient did not respond immediately, dose of glucocorticoid could be upregulated to $1.0 \mathrm{~g} / \mathrm{d}$ intravenous methylprednisolone (13). For patients with fatal myocarditis, the dosage of glucocorticoid still lacks consensus. Limited reports and researches supported a trend that there was a lower major adverse cardiac events rate with higher starting doses of immunosuppression (4). The dose of steroids should be gradually reduced after 3-5 days, tapering over one month and even longer in some severe cases (9). Both of our patients were treated with corticosteroids while the first patients received a higher dose of methylprednisolone and a slower course of dose reduction. It seemed that both patients responded well initially, but the second patient was admitted to hospital again afterwards, indicating a higher-dose of methylprednisolone may lead to better prognosis. Other immunosuppressive therapy such as intravenous immunoglobulins and/or plasma exchange also should be considered $(14,15)$. The patients in our report also overlapped other irAEs, including myasthenia gravis, hepatitis and hypothyroidism. In a report of 101 cases of ICI-related myocarditis carried out by Moslehi et al., 42\% of cases were concurrent with other severe irAEs, most commonly were myositis $(25 \%)$ and myasthenia gravis (11\%) (5). Johnson et al. demonstrated that myasthenia gravis presenting with myocarditis or myositis had higher mortality compared with myasthenia gravis alone (16). In particular, myasthenia gravis caused by PD-1 inhibitors appears to have some unique characteristics. It has been reported that myasthenic crisis or respiratory failure 
requiring ventilatory support in particular was common and occurred in nearly half of cases (17). Early identification of these conditions is needed.

\section{Conclusions}

Myocarditis is an uncommon but potentially fatal complication during the treatment with ICIs. Serum level of cardiac biomarkers, ECG, echocardiography, and cardiac imaging are valuable tools for the diagnosis of ICI-induced myocarditis. Patients usually can benefit from high-dose corticosteroid, but extra attention should be paid when patients presented with other irAEs like MG.

\section{Acknowledgments}

Funding: This work was supported by Clinical Plateau Discipline Project in Shanghai Pudong New Area (PWYgy2018-06).

\section{Footnote}

Reporting Checklist: The authors have completed the CARE reporting checklist. Available at: http://dx.doi.org/10.21037/ apm-20-2620

Peer Review File: Available at: http://dx.doi.org/10.21037/ apm-20-2620

Conflicts of Interest: All authors have completed the ICMJE uniform disclosure form (available at: http://dx.doi. org/10.21037/apm-20-2620). The authors have no conflicts of interest to declare.

Ethical Statement: The authors are accountable for all aspects of the work in ensuring that questions related to the accuracy or integrity of any part of the work are appropriately investigated and resolved. All procedures performed in studies involving human participants were in accordance with the ethical standards of the institutional and/or national research committee(s) and with the Helsinki Declaration (as revised in 2013). Written informed consent was obtained from the patient.

Open Access Statement: This is an Open Access article distributed in accordance with the Creative Commons Attribution-NonCommercial-NoDerivs 4.0 International License (CC BY-NC-ND 4.0), which permits the non- commercial replication and distribution of the article with the strict proviso that no changes or edits are made and the original work is properly cited (including links to both the formal publication through the relevant DOI and the license). See: https://creativecommons.org/licenses/by-nc-nd/4.0/.

\section{References}

1. Pardoll DM. The blockade of immune checkpoints in cancer immunotherapy. Nat Rev Cancer 2012;12:252-64.

2. Martins F, Sofiya L, Sykiotis GP, et al. Adverse effects of immune-checkpoint inhibitors: epidemiology, management and surveillance. Nat Rev Clin Oncol 2019;16:563-80.

3. Wang DY, Salem JE, Cohen JV, et al. Fatal Toxic Effects Associated With Immune Checkpoint Inhibitors: A Systematic Review and Meta-analysis. JAMA Oncol 2018;4:1721-8.

4. Mahmood SS, Fradley MG, Cohen JV, et al. Myocarditis in Patients Treated With Immune Checkpoint Inhibitors. J Am Coll Cardiol 2018;71:1755-64.

5. Moslehi JJ, Salem JE, Sosman JA, et al. Increased reporting of fatal immune checkpoint inhibitor-associated myocarditis. Lancet 2018;391:933.

6. Neilan TG, Rothenberg ML, Amiri-Kordestani L, et al. Myocarditis Associated with Immune Checkpoint Inhibitors: An Expert Consensus on Data Gaps and a Call to Action. Oncologist 2018;23:874-8.

7. Heinzerling L, Ott PA, Hodi FS, et al. Cardiotoxicity associated with CTLA4 and PD1 blocking immunotherapy. J Immunother Cancer 2016;4:50.

8. Johnson DB, Balko JM, Compton ML, et al. Fulminant Myocarditis with Combination Immune Checkpoint Blockade. N Engl J Med 2016;375:1749-55.

9. Sławiński G, Wrona A, Dąbrowska-Kugacka A, et al. Immune Checkpoint Inhibitors and Cardiac Toxicity in Patients Treated for Non-Small Lung Cancer: A Review. Int J Mol Sci 2020;21:7195.

10. Saibil SD, Bonilla L, Majeed H, et al. Fatal myocarditis and rhabdomyositis in a patient with stage IV melanoma treated with combined ipilimumab and nivolumab. Curr Oncol 2019;26:e418-21.

11. Hanneman K, Kadoch M, Guo HH, et al. Initial Experience With Simultaneous 18F-FDG PET/MRI in the Evaluation of Cardiac Sarcoidosis and Myocarditis. Clin Nucl Med 2017;42:e328-34.

12. Nensa F, Kloth J, Tezgah E, et al. Feasibility of FDG-PET in myocarditis: Comparison to CMR using integrated PET/MRI. J Nucl Cardiol 2018;25:785-94. 
13. Brahmer JR, Lacchetti C, Schneider BJ, et al. Management of Immune-Related Adverse Events in Patients Treated With Immune Checkpoint Inhibitor Therapy: American Society of Clinical Oncology Clinical Practice Guideline. J Clin Oncol 2018;36:1714-68.

14. Kostine M, Finckh A, Bingham CO, et al. EULAR points to consider for the diagnosis and management of rheumatic immune-related adverse events due to cancer immunotherapy with checkpoint inhibitors. Ann Rheum Dis 2021;80:36-48.

15. Balanescu DV, Donisan T, Palaskas N, et al.

Cite this article as: Chen Y, Jia Y, Liu Q, Shen Y, Zhu H, Dong X, Huang J, Lu J, Yin Q. Myocarditis related to immune checkpoint inhibitors treatment: two case reports and literature review. Ann Palliat Med 2021;10(7):8512-8517. doi: 10.21037/apm20-2620
Immunomodulatory treatment of immune checkpoint inhibitor-induced myocarditis: Pathway toward precisionbased therapy. Cardiovasc Pathol 2020;47:107211.

16. Johnson DB, Manouchehri A, Haugh AM, et al. Neurologic toxicity associated with immune checkpoint inhibitors: a pharmacovigilance study. J Immunother Cancer 2019;7:134.

17. Kao JC, Brickshawana A, Liewluck T. Neuromuscular Complications of Programmed Cell Death-1 (PD-1) Inhibitors. Curr Neurol Neurosci Rep 2018;18:63. 